\title{
UAV based on RTK high precision inspection system
}

\author{
Linxin $\mathrm{Li}$ \\ School of Electrical \& Electronic Engineering, North China Electric Power University, Baoding, \\ 071003 China \\ lilinxin75@gmail.com
}

Keywords: Unmanned aerial vehicle (UAV), real time kinematic (RTK), Intelligent rapid mission planning.

\begin{abstract}
RTK real-time dynamic relative positioning is to be able to get cm-level real-time positioning accuracy in the field of measuring methods, it USES the Real-time kinematic,RTK method, is a major milestone in GPS application, it appeared as the project lofting, topographic mapping, brought new dawn various control survey, GPS positioning accuracy from $\mathrm{m}$ level increased to cm level, the efficiency of field is improved greatly.
\end{abstract}

\section{Introduction:}

GPS global positioning system (GPS) is the United States armed forces joint development of satellite navigation system, can provide all kinds of users with precision of three-dimensional coordinates, speed and time, because of its high degree of automation, speed, high positioning accuracy, the economic benefit is remarkable, and many other advantages, is widely used.

Using GPS technology not only can build all kinds of precision control network and engineering control network, GPS technology also has the theodolite, total station and other traditional methods can't achieve the function and application field.At the same time of GPS - RTK technology has a cm-level real-time positioning accuracy, is currently in electric power, forestry, water conservancy, road and demarcation of measurement, and other fields has been very good applications.And applying RTK technology to transmission line measurement, especially the alignment, distance, height measurement, cross section andtransmission towerpositioning measurement, etc., can greatly improve the work efficiency and survey precision.

\section{Technical specifications}

RTK real-time dynamic relative positioning, generally consists of a base station and multiple rover real-time data processing is given by the ground station centimeter level and high precision positioning results, to achieve reliable fixed position precision, rover observation at least four satellites.

The rapid and high precision of GPS has good prospects for development, is also a hot issue in current research.Contains it involves the elaboration of stochastic models of GPS carrier phase observation quantity, GPS rapid positioning of ill-condition equation parameters estimation method, the ambiguity search and inspection of the cycle, and many other theoretical issues.At home and abroad to improve the positioning accuracy and positioning speed, adopt the method of establish a base station, the base station position are precisely known, by GPS post-processing software (such as RTKLIB) comprehensive error modeling, eliminate decreasing and eliminating error when the rover and base station distance of not more than $15 \mathrm{~km}$, track the error, troposphere delay, ionospheric delay error of the influence of residual error are less affected.

\section{Main research thinking and main research content}

Because of our research is four rotor unmanned aerial vehicle (UAV) for transmission lines in the inspection work, so the key research direction in the GPS data back, RTK processing, high precision 
position comes back.The design of the unmanned aerial vehicle (UAV) checking logic diagram below:

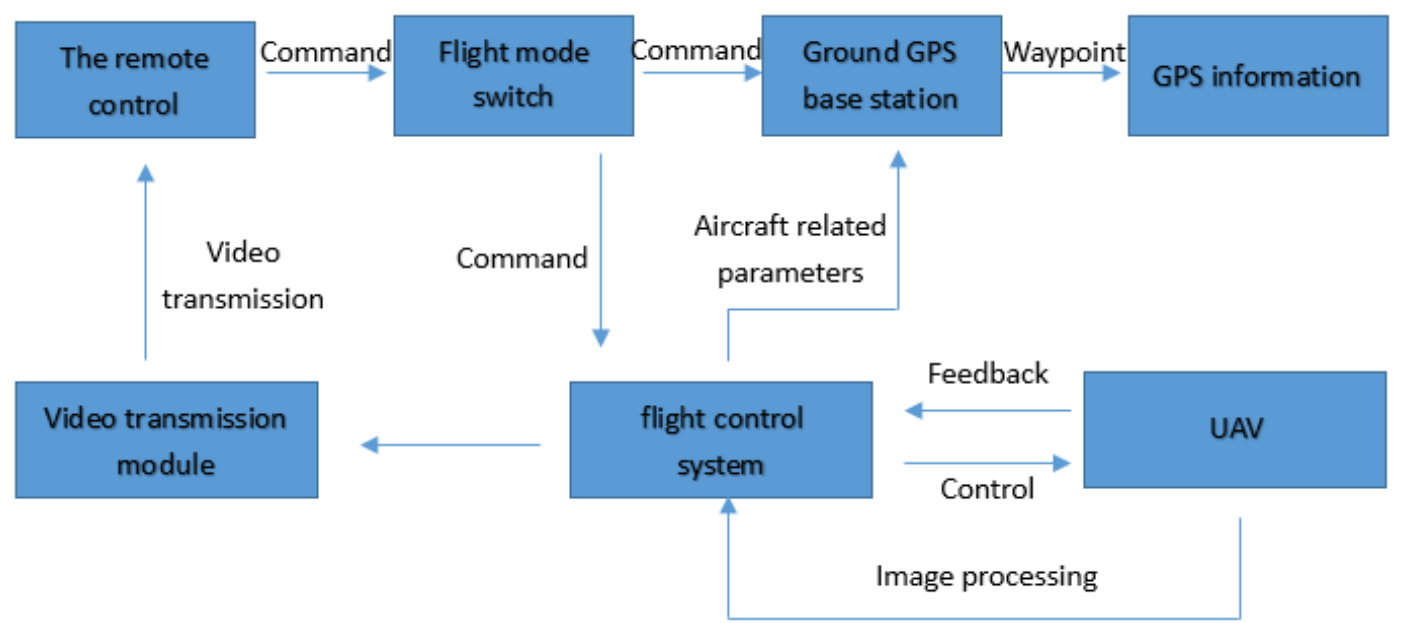

Fig. 1 The logic diagram of uav intelligent inspection system

By a flight control ground station software and the matching of the UAV flight control system implementation, through wireless data exchange between air and ground station, wireless data transmission adopts the GFSK modulation method, don't change any agreement and data users, to complete the function of wireless data transmission. Frequency $433 \mathrm{MHZ}$ (exemption) application frequency transmission distance above $1000 \mathrm{~m}$, open without interference can be up to $1.5 \mathrm{Km}$, air baud rate 115200 bps to meet the requirement of data exchange. In the Arduino development environment for UAV flight control system development, the Arduino program in a programming language to microcontroller, can compile a binary stream file, and then you can burn into the Arduino microcontroller. Based on the wiring of the Arduino programming language, and based on the Arduino integrated development environment to implement the Arduino controller programming.In the Arduino program, allowing only have the Arduino language code, and also allows the Arduino and other language such as C, Java, such as writing some software, the synchronization between them Such as Processing, such as Flash, MaxMSP communication mode.

AVR series controller in terms of price and function used to implement the Arduino microprocessor controller is the best choice, with USB interface for power supply, do not need an external power supply, of course, also can use external 9 v DC power input.

Arduino more powerful support is burn online, ISP when allowed to burn "bootloader" firmware to AVR chip. After burn bootloader, can through the USB to Rs232 line or a serial port to update the firmware.

Design plan in unmanned aerial vehicle (UAV) with GPS module, the module through a five terminals and I2C port connected to the flight control plate, the real-time data exchange, location in the program of flight control the underlying data signals in a flash, flash other subroutine calls g_GPS data, in-depth development based on the case does not affect the normal operation of the whole system to improve the accuracy of positioning signal processing, and external interference in a timely switch back to the on-board GPS to ensure normal flight of UAV.

Only a single digital are connected to the PC through a serial port, we plan to take under such restrictions from data capture to RTK GPS data signal difference, as above mentioned RTKLIB and ground station can not used in the realization of serial port called directly, through research and theoretical speculation, to determine the file as an intermediate amount to communication the two software.

RTKLIB floor between the base station and rover data to improve the positioning accuracy of the results, determine the base station directly connected to the PC on the ground, but the rover data exchange due to serial portrestrictions, we adopted virtual serial port to intercept the signal and real-time exchange RTKLIB, then the location of the high precision data obtained from the returned by digital flight control, but the data doesn't directly by flight control side subroutine program calls. 


\section{Development process}

The overall operation of the process as shown:

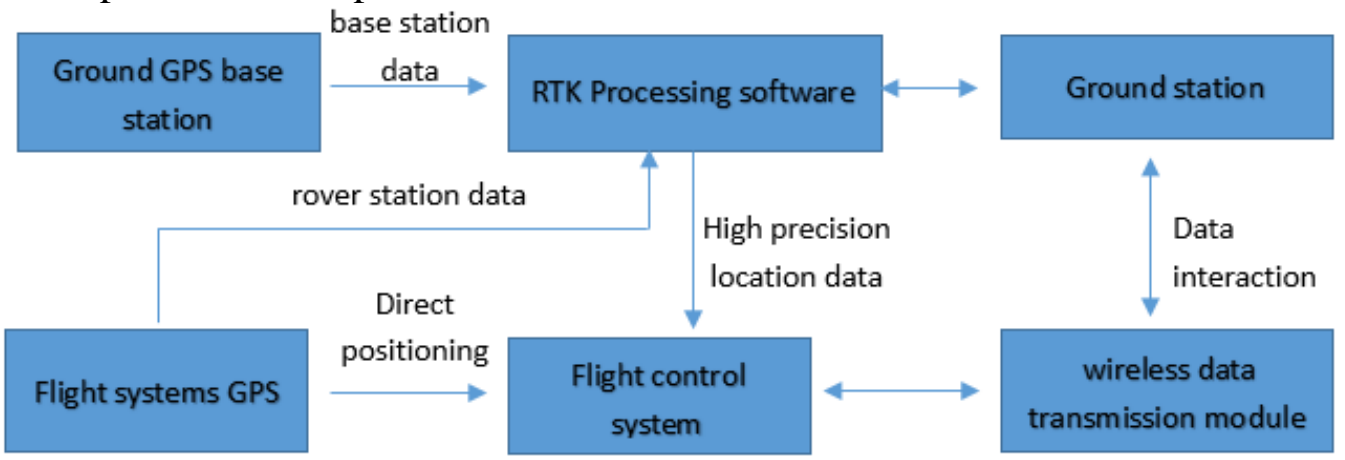

Fig. 2 overall operation of the process

By wireless data transmission and ground station to complete the system and eventually improve the positioning accuracy of the results, through measuring single GPS positioning accuracy is $1.2 \mathrm{~m}$, portable single base station positioning system eventually can realize cm-level positioning accuracy. Meet high factor of safety inspection for overhead transmission circuits.

With the development of current science and technology, more data for transmission line shape and position requirements, can be transmission tower as RTK positioning base station, about 10 to 15 $\mathrm{km}$ to establish a source point, on the one hand can achieve much higher precision of base station positioning, on the other hand for the virtual model of the power grid construction, provide data support real-time monitoring.

\section{Summary}

RTK positioning technology has many advantages in power transmission line inspection, according to the current national ultra-high-voltage electricity lines construction and the reform of power grid, combined with the combined use of UAV it has a very broad prospect. Using UAV combined with RTK technology is a great breakthrough in the measurement for overhead power transmission line inspection, the technology will be in overhead transmission way erection, engineering surveying, play an important role in the daily inspection, etc.

\section{Reference}

[1] Zhang Qin, Li right .GPS measuring principle and application [M] Beijing: Science Press, 2005, 134-136.

[2] EI-MOWAFY A.Analysis of the Design Parameters of Multi-reference Station RTK GPS Networks [J]. Journal of Satellite and Land Information Science (SaLIS), 2005, 65 (1): 17-26.

[3] T.Takasu.RTKLIB Ver.2.4.2 Manual.April 29, 2013. http:/www.RTKLIB.com/prog/ manual_2.4.2.pdf.

[4] Tao Li, Jinling Wang. Analysis of the upper bounds foe the integer ambiguity validation statistics [J]. GPS Solution, 2013, DOI 10.1007/s10291-013-0312-1. 